\title{
Use of Over-The-Counter Cough and Cold Medications in the Pediatric Population: An Overview and Recommendations
}

\author{
Zeina M Saleh*, Mohammed M Saleh and Fadl A Zeineddine \\ American University of Beirut, Lebanon
}

Submission: February 13, 2017; Published: February 28, 2017

*Corresponding author: Zeina M Saleh, MD American University of Beirut, Lebanon, Email: zms19@aub.edu.lb

\section{Mini Review}

The common cold is a common illness that burdens all age groups, normally affecting children around 6 times a year and adults two to four times a year [1]. Signs and symptoms include fever, cough, sore throat, and rhinorrhea. Patients and their parents often seek medications such as antibiotics and cough and cold medications [2]. While antibiotics are prescription drugs, over-the-counter (OTC) cough and cold medications (CCM) are readily available and frequently purchased. Such medications include 1 or more of the following: antihistamines, decongestants, expectorants, or antitussives $[3,4]$.

In 1976, OTC CCM use was permitted in the pediatric age group by the FDA as they were generally considered safe medications, however, there were no systematic analyses regarding the safety and tolerability of these drugs. Reported side effects associated with these medications included tachycardia, excitability, blurry vision, agitation, altered levels of consciousness, and respiratory depression. Decades later tens of children were reported dead due to the use of these medications [4,5]. Deaths were attributed to incorrect dosages, unintentional congestion, and improper attention to medication ingredients leading to overdoses $[3,5]$. These medications, despite their risk, were widely used. Two surveys aimed at measuring the prevalence revealed that approximately $10 \%$ of the children were taking OTC CMM in a week. With increasing deaths and side effects reported, the FDA started an investigation that led to recommendations warning against the use of OTC CCM in children less than 2 years old in early 2008. Later that year, the Consumer Healthcare Products Association extended the age limit to include all children less than 4 years of age, an extension that was supported by the FDA $[5,6]$.

Whilst investigating the safety of OTC CMM, researchers studied the efficacy of these products in parallel. Among the earliest studies that addressed the efficacy of medications included antihistamines combined with decongestants. Patients, whose ages ranged from 6 months to 6 years, were randomized

into a medication group, a placebo group, and a no medication group. Results revealed that both the medication group and placebo group had similar improvement. Similarly, other drugs were tested, and multiple studies including a meta-analyses in the Cochrane Collaboration, proved and concluded that when comparing the use of OTC CMM versus placebo in children with the common cold, there was no difference in resolution of symptoms, further solidifying previous studies that questioned the efficacies of these drugs [5-7].

Despite FDA warnings and labeling changes, the use of OTC CMM in children was and most likely still continues to be significant. According to the FDA 860 parents out of 1000 purchase the medications instead of or prior to consulting with their pediatricians. The ongoing safety risk coupled with the highly prevalent use of these drugs led the FDA in 2015 to release a formal warning against using any form of OTC CCM in all children under age 18 [8].

Due to the risk this imposes on the pediatric population, attempts to raise awareness might help decrease morbidity and mortality. Healthcare providers are the pillars of raising such awareness as parents often rely on their advice. Pediatricians should encourage the use of antipyretics as well as nonmedical treatments such as honey (except in children less than 1 year of age), saline nasal washes, and proper hydration. Prevention should also be addressed during visits, including avoiding sick contacts and breastfeeding as it provides the child with immunity. However, given the fact that many parents do not seek medical advice for the common cold, pharmacist can also play a vital role in reducing medication side effects by properly educating the parents about dosages and preventing purchase of medications with overlapping ingredients to avoid toxicity. Additionally, parents are encouraged to read labels, instructions, and ingredients prior to administering the medication. It is 
also strongly recommended that such medications stay out of reach of children to prevent unintentional congestion [2,5]. Drug manufacturing companies can also aid in preventing unintentional congestion by making child-protected lids [2].

\section{Conclusion}

In conclusion, as there is no proof of the efficacy of OTC CMM and ample proof of the safety risk associated with their use, it is therefore recommended that the use of OTC CCM be avoided in children less than 18 years old, and alternative managements be encouraged.

\section{References}

1. https://www.iqwig.de/en/home.2724.html

2. Garbutt JM, Sterkall R, Banister C, Walbert C, Robert R (2010) Physician and parent response to the FDA advisory about use of over-the-counter cough and cold medications. Acad Pediatr 10(1): 64-69.
3. Lazarus SG, Lanski SL, Smith AS, Simon HK (2013) Cold Preparation Use in Young Children After FDA Warnings: Do Concerns Still Exist? Clinical Pediatrics 52(6): 534-539.

4. Chandelia S, Dhankar M, Salhan M (2015) Pediatrician's cough and cold medication prescription for hypothetical cases - A cross-sectional multi-centric study. Saudi Pharmaceutical Journal 24(2): 176-181.

5. Briars LA (2009) The Latest Update on Over-the-Counter Cough and Cold Product Use in Children. J Pediatr Pharmacol Ther 14(3): 127-131.

6. Spring S (2008) Using over-the counter cough and cold products in children. Food and Drug Administration.

7. Shefrin AE, Goldman RD (2009) Use of over-the-counter cough and cold medications in children. Can Fam Physician 55(11) 1081-1083.

8. FDA. (2016, April 11). Use Caution When Giving Cough and Cold Products to Kids. Retrieved from https://www.fda.gov/Drugs/ ResourcesForYou/SpecialFeatures/ucm263948.htm 